\title{
A Lattice Boltzmann Model for Multi-phase Fluid Flows
}

\author{
Daryl Grunau ${ }^{1,3}$, Shiyi Chen ${ }^{2}$, and Kenneth Eggert ${ }^{1}$ \\ ${ }^{1}$ Earth and Environment Science Division, Los Alamos National Laboratory, Los Alamos, NM 87545 \\ ${ }^{1}$ Theoretical Division and Center for Nonlinear Studies, Los Alamos, NM 87545 \\ ${ }^{3}$ Department of Mathematics, Colorado State University, Fort Collins, CO 80523
}

\begin{abstract}
We develop a lattice Boltzmann equation method for simulating multi-phase immiscible fluid flows with variation of density and viscosity, based on the model proposed by Gunstensen et al 9] for two-component immiscible fluids. The numerical measurements of surface tension and viscosity agree well with theoretical predictions. Several basic numerical tests, including spinodal decomposition, two-phase fluid flows in two-dimensional channels and two-phase viscous fingering, are shown in agreement of experiments and analytical solutions.
\end{abstract}




\section{Introduction}

It has recently been shown that lattice gas computational fluid dynamics, including lattice gas automata (LGA) [1] and lattice Boltzmann (LB) [2] equation methods, provide alternative numerical techniques for solving the Navier-Stokes equations, multi-

phase fluid flows [3, 4, 5] and a variety of other fluid systems [6, 7]. The parallel nature of these newly developed schemes, adapted from cellular automata, affords an easy implementation of fast, efficient and accurate simulations on parallel machines.

To solve the incompressible fluid equations by traditional numerical methods such as finite differences or finite elements, one must deal with a Poisson equation for the pressure term that is induced by the continuum condition and the momentum equation. This fact has brought to light a crucial difference between lattice gas methods and traditional algorithms, since a lattice gas solver is only required to solve the kinetic equation of the particle distribution. All other quantities, including density, velocity and energy can be obtained by a macroscopic averaging process through the distribution function. Therefore, the pressure effects on the momentum equation are controlled by an equation of state.

Strictly speaking, lattice gas automata should be restricted to solving compressible fluid flows. The incompressible limit theory of lattice gas methods [8] and their numerical simulations [7], however, predict and demonstrate that such a lattice gas solution at low Mach number produces results comparable with those of the incompressible Navier-Stokes equations for a wide variety of problems and with very small numerical errors. Also, two-phase surface dynamic boundaries and wall boundaries are far more easily implemented with a lattice gas method compared to direct simulations of the incompressible Navier-Stokes equations [3, 7].

Rothman and Keller[3] were the first to extend the single-phase lattice gas model 
proposed by Frisch, Hasslacher and Pomeau[1] to simulate multi-phase fluid problems. Colored particles were introduced to distinguish between phases, and a nearestneighbor particle interaction was used to facilitate interfacial dynamics, such as Laplace's formula for surface tension. Later, Somers and Rem[4], and Chen et al[5] extended the original colored particle scheme by introducing colored holes. It has been shown[5] that the colored-hole lattice gas method extends the original nearest neighbor particle interaction to several lattice lengths, leading to a Ukowa potential. Moreover, the colored-hole scheme carries purely local information in it's particle collision step, reducing the size of the look-up table in the algorithm and consequently speeding up the simulation.

Although two-phase lattice gas algorithms are able to produce interesting surface phenomena, they are difficult to compare quantitatively with experiments and other numerical simulations due to their noisy characteristics induced by particle fluctuations. The lattice Boltzmann model proposed by McNamara and Zanetti[2], however, solves the kinetic equation for the particle distribution instead of tracking each particle's motion, as is done in lattice gas methods. Nonetheless, lattice gas algorithms will be superior to lattice Boltzmann models for simulating underlying physics and modeling microscopic dynamics such as correlation effects and phase transitions, since they contain more information about the microscopic behavior of particles. On the other hand, to provide a numerical method for solving partial differential equations governing macroscopic behavior, lattice Boltzmann schemes will be at least as good as lattice gas models. The finite-difference nature of lattice Boltzmann methods not only can simulate the macroscopic equations more efficiently and accurately, but also preserve some of the advantages of lattice gas models, such as their parallel computing nature and their ease of boundary implementation.

Combining the lattice Boltzmann model of McNamara and Zanetti[2] and the 
two-phase lattice gas model of Rothman et al, Gunstensen et al [9] proposed a lattice Boltzmann method for solving two-phase fluid flows. An important contribution of this model is the introduction of a perturbation step (shown in detail below) so that Laplace's formula at an interface can be approximately recoved. This lattice Boltzmann method has been used in several applications [10, however it has a few fundamental problems. First, the model does not solve the exact two-phase fluid equations - although Galilean invariance is recovered by the proper assignment of rest particles, the equation of state remains to be velocity dependent [11]. Secondly, the model uses a fully linearized collision operator, which involves a $24^{*} 24$ matrix multiplication at each time step and position in three dimensional space, reducing computational efficiency. Finally, the model is restricted to two-phase fluid flows having the same densities and viscosities. In this paper, we extend their model by using a single-time relaxation lattice Boltzmann model. With the proper choice of the particle equilibrium distribution function, we are able to recover the incompressible Navier-Stokes equations for the color-blind fluid. In addition, our new model makes provision for variable densities and viscosities by use of the freedom of the rest particle equilibrium distribution, and a space dependent relaxation process. For simplicity we present a two-phase, two-dimensional fluid model on a hexagonal lattice in this paper. The extension of the current model to multi-phase fluids and other lattices, including a two-dimensional square lattice, a three-dimensional Face-Centered Hypercubic lattice [8] and a Body-Centered-Cubic [12] lattice, will be presented in detail in another paper 13.

\section{Numerical Model}

Denote $f_{i}(\mathbf{x}, t), f_{i}^{(r)}(\mathbf{x}, t)$ and $f_{i}^{(b)}$ as the particle distribution functions at space $\mathbf{x}$ and time $t$ for total, red, and blue fluids respectively. Here $i=0,1, \cdots, N$, where $N$ is the 
number of moving particle directions (6 for the hexagonal lattice and 8 for the square lattice in two dimensions), and $f_{i}=f_{i}^{(r)}+f_{i}^{(b)}$. The lattice Boltzmann equation for both red and blue fluids can be written as follows:

$$
f_{i}^{k}\left(\mathbf{x}+\mathbf{e}_{i}, t+1\right)=f_{i}^{k}(\mathbf{x}, t)+\Omega_{i}^{k}(\mathbf{x}, t),
$$

were $k$ denotes either the red or blue fluid, and $\Omega_{i}^{k}=\left(\Omega_{i}^{k}\right)^{1}+\left(\Omega_{i}^{k}\right)^{2}$ is the collision operator. Note that in the two-phase lattice Boltzmann model by Gunstensen et al only a color-blind kinetic equation was given. The first term of the collision operator, $\left(\Omega_{i}^{k}\right)^{1}$, represents the process of relaxation to local equilibrium. For simplicity, we use a linearized collision operator with a single time relaxation parameter $\tau_{k}$ 14,

$$
\left(\Omega_{i}^{k}\right)^{1}=\frac{1}{\tau_{k}}\left(f_{i}^{k}-f_{i}^{k(e q)}\right) .
$$

Here $f_{i}^{k(e q)}$ is the local equilibrium state depending on the local density and velocity, and $\tau_{k}$ is the characteristic relaxation time for species $k$. In lattice gas automaton models, the form of the local equilibrium state and rate of relaxation to the local equilibrium distribution are completely determined by the particle scattering process. From a statistical point of view, the macroscopic effects of these collisions only arise in the transport coefficients. Therefore, lattice Boltzmann models ignore particle collision details such as collision cross sections and frequencies. In addition, the local equilibrium state can be arbitrarily chosen[11], with the exception that it must satisfy the conservation of mass and momentum:

$$
\begin{aligned}
& \rho_{r}=\sum_{i} f_{i}^{r}=\sum_{i} f_{i}^{r(e q)}, \\
& \rho_{b}=\sum_{i} f_{i}^{b}=\sum_{i} f_{i}^{b(e q)},
\end{aligned}
$$

and

$$
\rho \mathbf{v}=\sum_{i, k} f_{i}^{k} \mathbf{e}_{i}=\sum_{i, k} f_{i}^{k(e q)} \mathbf{e}_{i}
$$


Here $\rho_{r}$ and $\rho_{b}$ are densities of the red and blue fluids respectively, $\rho=\rho_{r}+\rho_{b}$ is the total density and $\mathbf{v}$ is the local velocity.

Following the pressure-corrected Galilean-invariant single-phase lattice Boltzmann model proposed by reference [11], we assume the following equilibrium distribution for both red and blue fluids:

$$
\begin{aligned}
f_{i}^{r(e q)} & =\rho_{r} /\left(6+m_{r}\right)+\rho_{r}\left(\mathbf{e}_{i} \cdot \mathbf{v} / 3+2 / 3\left(\mathbf{e}_{i}\right)_{\alpha}\left(\mathbf{e}_{i}\right)_{\beta} v_{\alpha} v_{\beta}-\frac{1}{6} \mathbf{v}^{2}\right), \\
f_{0}^{r(e q)} & =\rho_{r} m_{r} /\left(6+m_{r}\right)-\rho_{r} \mathbf{v}^{2} \\
f_{i}^{b(e q)} & =\rho_{b} /\left(6+m_{b}\right)+\rho_{b}\left(\mathbf{e}_{i} \cdot \mathbf{v} / 3+2 / 3\left(\mathbf{e}_{i}\right)_{\alpha}\left(\mathbf{e}_{i}\right)_{\beta} v_{\alpha} v_{\beta}-\frac{1}{6} \mathbf{v}^{2}\right), \\
f_{0}^{b(e q)} & =\rho_{b} m_{b} /\left(6+m_{b}\right)-\rho_{b} \mathbf{v}^{2} .
\end{aligned}
$$

Here we have introduced two parameters, $m_{r}$ and $m_{b}$. They can be understood as the number of degenerate rest particle states for the red and blue fluids respectively. To achieve a stable interface, we furthermore assume that the moving particle distributions for both red and blue fluids are the same when $\mathbf{v}=0$ :, i.e., $d=\rho_{r} /\left(6+m_{r}\right)=\rho_{b} /\left(6+m_{b}\right)$. This implies the following density ratio: $\frac{\rho_{r}}{\rho_{b}}=\frac{6+m_{r}}{6+m_{b}}$.

The second part of the collision operator is similar to that given in Gunstensen et al's paper:

$$
\left(\Omega_{i}^{k}\right)^{2}=\frac{A_{k}}{2}|\mathbf{F}|\left(\left(\mathbf{e}_{i} \cdot \mathbf{F}\right)^{2} /|\mathbf{F}|^{2}-1 / 2\right),
$$

where $\mathbf{F}$ is the local color gradient, defined as:

$$
\mathbf{F}(\mathbf{x})=\sum_{i} \mathbf{e}_{i}\left(\rho_{r}\left(\mathbf{x}+\mathbf{e}_{i}\right)-\rho_{b}\left(\mathbf{x}+\mathbf{e}_{i}\right)\right)
$$

Note that in a single-phase region of our incompressible fluid model, $\mathbf{F}=0$. Thus the second term of the collision operator, $\left(\Omega_{i}^{k}\right)^{2}$, only has contribution at two-phase interfaces. The parameter $A_{k}$ is a free parameter, controlling the surface tension (shown before). For simplicity, we choose $A_{r}=A_{b}$ in this paper.

To obtain a surface tension, we follow Rothman's scheme[3] to redistribute colored 
particles at two-phase interfaces (without changing the total particle distribution, $f_{i}$ ) by enforcing the red color momentum $\mathbf{j}^{r}=\sum_{i} f_{i}^{r} \mathbf{e}_{i}$, to align with the direction of the local color gradient. In other words, we redistribute the red density at an interface to maximize the following quantity:

$$
-\left(\mathbf{j}^{r} \cdot \mathbf{F}\right) .
$$

The blue particle distribution can then be recovered using: $f_{i}^{b}=f_{i}-f_{i}^{r}$.

To derive hydrodynamics, we use a long-wavelength, low-frequency approximation and a multiscaling analysis as follows:

$$
\begin{gathered}
\frac{\partial}{\partial t}=\epsilon \frac{\partial}{\partial t_{1}}+\epsilon^{2} \frac{\partial}{\partial t_{2}}+\ldots, \\
\frac{\partial}{\partial x}=\epsilon \frac{\partial}{\partial x_{1}} .
\end{gathered}
$$

Here $t_{1}$ and $t_{2}$ represent fast and slow time scales respectively, and $\epsilon$ is assumed to be a small expansion parameter.

A Taylor series expansion of Equation (臬) to second order in the lattice spacing and time step gives the following continuum kinetic equation:

$$
\frac{\partial f_{i}^{k}}{\partial t}+\mathbf{e}_{i} \cdot \nabla f_{i}^{k}+\frac{1}{2} \mathbf{e}_{i} \mathbf{e}_{i}: \nabla \nabla f_{i}^{k}+\mathbf{e}_{i} \cdot \nabla \frac{\partial}{\partial t} f_{i}^{k}+\frac{1}{2} \frac{\partial}{\partial t} \frac{\partial}{\partial t} f_{i}^{k}=\Omega_{i}^{k} .
$$

Taking the zero and first order moments of $\mathbf{e}_{i}$ over equation (2), we readily obtain the continuum equations for the red and blue fluids, and the following momentum equation for the color-blind (total) fluid:

$$
\frac{\partial\left(\rho v_{\alpha}\right)}{\partial t}+\sum_{i, k} \mathbf{e}_{\alpha} \mathbf{e}_{i}\left(f_{i}^{k(e q)}+f_{i}^{k(1)}\right)=0
$$

where $f_{i}^{k(1)}$ is the next order perturbation for $f_{i}^{k}$. Note that since

$$
\sum_{i}\left(\Omega_{i}^{k}\right)^{2}=\sum\left(\Omega_{i}^{k}\right)^{2} \mathbf{e}_{i}=0
$$


and

$$
\mathbf{F}=\frac{1}{3} \nabla\left(\rho_{r}-\rho_{b}\right)+\cdots \sim \epsilon+o\left(\epsilon^{2}\right),
$$

the second term in the collision operator does not contribute to the continuum and momentum equations of the first order approximation. It, however, will contribute to the pressure term at an interface: $P=P_{0}+\epsilon|\nabla F|$, where $P_{0}$ comes from the equation of state.

Our two-phase immiscible fluid model contains three fluid regions: a red or blue homogeneous region and a thin region where the two fluids mix at the interfaces. In a homogeneous region, the evolution of our model will recover the Navier-Stokes equations with viscosity of $\nu_{k}=\left(2 \tau_{k}-1\right) / 8$ and sound speed of $c_{k}=\sqrt{3 /\left(6+m_{k}\right)}$ according to the Chapman-Enskog expansion shown in reference [12]. A variation of viscosities for the two fluids can be obtained by choosing different $\tau_{k}$.

The last issue remaining to be addressed is the interfacial dynamics that take place in a region where red and blue fluids are adjacent. Usually the thickness of such an interface will depend on an averaged relaxation time, $2 \tau_{r} \tau_{b} /\left(\tau_{r}+\tau_{b}\right)$, and the rest particle distribution. There are several ways to construct a relaxation parameter so that the red and blue fluids have a smooth change of viscosity at their interfaces. To do this, we define an order parameter, $\psi$, depending on red and blue densities as follows: $\psi=\frac{\rho_{r}-\rho_{b}}{\rho_{r}+\rho_{b}}$. Note that in general $|\psi| \leq 1$, however in a purely red fluid region, $\psi=1$, and in a purely blue fluid region, $\psi=-1$. To continuously connect relaxation parameters $\tau_{r}$ and $\tau_{b}$ at an interface, we employ the following simple formula:

$$
\tau= \begin{cases}\tau_{r} & \psi>\delta \\ g_{r}(\psi) & \delta \geq \psi>0 \\ g_{b}(\psi) & 0 \geq \psi \geq-\delta \\ \tau_{b} & \psi<-\delta\end{cases}
$$

where $g_{r}(\psi)$ and $g_{b}(\psi)$ are second order functions of $\psi$ :

$$
g_{r}(\psi)=\alpha+\beta \psi+\gamma \psi^{2}
$$


and

$$
g_{b}(\psi)=\alpha+\eta \psi+\xi \psi^{2}
$$

Assuming that $g_{r}(\delta)=\tau_{r}, g_{b}(-\delta)=\tau_{b}, \frac{\partial \tau}{\partial \psi}=0$ at $|\psi|=\delta$ and that $g_{r}(0)=g_{b}(0)$, we have: $\alpha=2 \tau_{r} \tau_{b} /\left(\tau_{r}+\tau_{b}\right), \beta=2\left(\alpha-\tau_{b}\right) / \delta, \gamma=\beta /(2 \delta), \eta=2\left(\tau_{r}-\alpha\right) / \delta$ and $\xi=\eta /(2 \delta)$. Here $\delta$ is a free parameter controlling the interface thickness, $\delta \leq 1$.

Using the standard definition of surface tension [9], $\sigma=\int\left(P_{n}-P_{t}\right) d x$, and after some algebra 13], we obtain a theoretical formula for the surface tension on a twodimensional hexagonal lattice: $\sigma=9 A<\tau>d\left(12+m_{r}+m_{b}\right)$, where $<\tau>$ is the averaged relaxation time step across the interface. Here the integration is over whole space along the direction perpendicular to a given interface, and $P_{n}$ and $P_{t}$ are respectively the normal and tangential stress tensor at the two-phase interface. For the interface relaxation interpolation scheme in this paper, we use $<\tau>=$ $2 \tau_{r} \tau_{b} /\left(\tau_{r}+\tau_{b}\right)$. In Fig. 1, we show the theoretical prediction $(-)$ and numerical measurements $(\diamond$ and + ) of $\sigma$ as a function of particle density $d$ with a mass ratio, $m_{r} / m_{b}=2$. The $\diamond$ symbols represent numerical measurements of surface tension using the above definition, and the + signs represent the surface tension obtained by Laplace's formula with measurements of the pressure drop across a bubble. As seen, the theoretical prediction and numerical measurements agree very well in both cases.

\section{Numerical Simulations and Discussion}

To demonstrate the application of the presented lattice Boltzmann model, we show three numerical examples in the following sections. These simulations have been carried out using the CM-200 at the Advanced Computing Laboratory at Los Alamos National Laboratory. The first numerical test is the study of the phase segregation process by two-dimensional spinodal decomposition when the fluids have differing densities. Fig. 2 is a snapshot $(t=8300)$ of the two-phase area distribution when 
the density ratio, $\gamma=\frac{m_{r}}{m_{b}}$, is 10 . One can see that the red fluid represents a high density region and the blue fluid represents a low density region. The initial condition for this simulation is constant density and random color distribution with periodic boundaries. Here the surface tension parameter is set to $A=0.01$. The current model preserves the basic two-phase segregation processes and, in simulations not shown here, we have seen the preservation of stable two-phase interfaces for much higher density ratios $(\sim 200)$. This capability will allow us to approach the simulation of gas and oil mixing flows.

The second numerical test is two-dimensional channel flow with two fluids having different viscosities. A lattice length of 65 in the y-direction $(W=65)$ and 128 in the $\mathrm{x}$-direction $(L=128)$ is used, with a non-slip condition on both the upper and bottom boundaries. Initially, the lower half space (from lattice 1 to 32 ) is filled with red fluid, and the top half space (from lattice 34 to 65 ) is filled with blue fluid. The middle line is initialized to half red and half blue, and the relaxation parameters for red and blue fluids are assigned values of $\tau_{r}=2$ and $\tau_{b}=1$. Using the viscosity formula discussed above, this translates into a kinematic viscosity ratio of 3:1 for the red and blue fluids respectively. A forcing technique, as described in reference [12], is used to establish a small pressure gradient across the length of the channel. Assuming a thin interface in the middle, an analytical solution of the velocity distribution as a function of $y$ can be derived, centered on the middle of the channel 15$]$ :

$$
\begin{aligned}
& v_{x}^{r}=\frac{\left(P_{0}-P_{L}\right) w^{2}}{2 \mu_{1} L}\left[\frac{2 \mu_{r}}{\mu_{r}+\mu_{b}}+\frac{\mu_{r}-\mu_{b}}{\mu_{r}+\mu_{b}} \frac{y}{w}-\frac{y}{w}^{2}\right], \\
& v_{x}^{b}=\frac{\left(P_{0}-P_{L}\right) w^{2}}{2 \mu_{2} L}\left[\frac{2 \mu_{b}}{\mu_{r}+\mu_{b}}+\frac{\mu_{r}-\mu_{b}}{\mu_{r}+\mu_{b}} \frac{y}{w}-\frac{y}{w}^{2}\right],
\end{aligned}
$$

where $\left(P_{0}-P_{L}\right) / L$ is the pressure gradient across the channel, $\mu_{r}$ and $\mu_{b}$ are shear viscosities for red (0.7875) and blue (0.2625) fluids respectively. Here also $w=\sqrt{3} \mathrm{~W} / 4$ is half the channel width, where the factor $\sqrt{3} / 2$ is a hexagonal lattice effect. In Fig. 
3 we present the analytical prediction $(-)$ and direct numerical simulation $(\diamond)$ for velocity as a function of channel width $y$. As seen, the agreement is excellent.

The last numerical test is a two dimensional Hele-Shaw viscous fingering experiment. The upper and lower walls are assigned a no-slip boundary condition, and the fluids are given a viscosity ratio of 1:10 by assigning $\tau_{r}=1$ and $\tau_{b}=5.5$. To develop a Hele-Shaw pattern, an initial perturbation is introduced at the interface 16, 17 which is then forced by maintaining a high pressure at the inlet and low pressure at the outlet. The shape of the finger can be altered by a change in the surface tension parameter $A$, given as 0.0065 in this experiment. Fig. 4 shows the evolution of a less viscous fluid penetrating one having a higher viscosity at times $=0,2000$, 4000, 6000, 8000 and 10000. We see that a stable finger develops and is maintained throughout the simulation, although we expect a tip-splitting instability to occur for smaller values of $A$, as demonstrated by reference [17]. This phenomenon will be discussed in more detail in a subsequent paper. The fluid patterns shown here are similar to results obtained by other numerical methods [16, 17].

The lattice Boltzmann model in this paper is the extension of the model proposed by Gunstensen et al. The current model gives an exact Navier-Stokes solution, in the incompressible limit, for each fluid individually. The surface tension at an interface satisfies the Laplace formula, and the model has the capability to simulate two-phase fluid flows with different densities and viscosities. The simulation results for several typical two-phase fluid flows are shown, in good agreement with analytical solutions and some experimental observations. Several issues, however, remain to be addressed. First, to achieve a density variation, the current model uses the freedom of the rest particle equilibrium distribution. Although the scheme is simple, it is difficult to obtain high velocity flows since the assignment of a high particle mass ratio will accumulate a large number of particles in the rest state, reducing the fluid speed. In 
addition, the equation of state of the current model is that of an ideal gas similar to the original FHP model. To simulate fluid flows with a large density variation, a lattice gas model with an equation of state for non-ideal gases (liquids) may provide some alternatives. Second, the current model uses two parabolic curves to match the interface relaxation characteristic time in the mixing region. For a very thin interface (for example, one lattice width), the parameter, $\delta$, will be negligible. On the other hand, for flows with slow relaxation, the interface will be much wider. The relaxation form and the choice of $\delta$ may affect interfacial dynamics. Other functional forms, such as a bi-normal distribution, may give better results.

We thank G. D. Doolen and Hudong Chen for useful suggestions. Eugene Loh has contributed to the original CM-2 code development. This work is supported by the US Department of Energy at Los Alamos National Laboratory. Numerical simulation were carried out using the computational resources at the Advanced Computing Laboratory at the Los Alamos National Laboratory.

\section{References}

[1] Frisch, U., Hasslacher B., and Y. Pomeau, "Lattice-Gas Automata for the NavierStokes Equation", Phys. Rev. Lett., 56 (1986):1505-1508.

[2] McNamara, G. G., and G. Zanetti, "Use of the Boltzmann Equation to Simulate Lattice-Gas Automata", Phys. Rev. Lett., 61(20) (1988):2332-2335.; Higuera, F. J., Succi, S. and R. Benzi, "Lattice Gas Dynamics with Enhanced Collisions" Europhys. Lett. 9(4) (1989):345-349.

[3] Rothman, D. H., and J. M. Keller, "Immiscible Cellular-Automaton Fluids", J. Stat. Phys., 52(3/4) (1988):1119-1127. 
[4] Somers, J. A., and P. C. Rem, "Analysis of Surface Tension in Two-Phase Lattice Gasses", Physica D, 47 (1991):39-46.

[5] Chen, S., Doolen, G. D., Eggert, K., Grunau, D., and E. Y. Loh, "Local latticegas model for immiscible fluids", Phys. Rev. A, 43(12) (1991):7053-7056.

[6] G. D. Doolen ed., Lattice Gas Methods for PDEs, Addison-Wesley, 1989; G. D. Doolen, ed. Lattice Gas Methods for PDE's: Theory, Applications and Hardware, Physica D, 47 No. 1 \& 2 (1991).

[7] Chen, S., Doolen, G. D. and W. H. Matthaeus, "Lattice Gas Automata for Simple and Complex Fluids," J. Stat. Phys., 64(5/6) (1991):1133-1162.; Kapral, R., Lawniczak, A. and P. Masiar, "Oscillations and Waves in a Reactive Lattice-Gas Automaton," Phys. Rev Lett. 66(19) (1991):2539-2542.

[8] Frisch, U., d'Humières, D., Hasslacher, B., Lallemand, P., Pomeau Y. and J. P. Rivet, "Lattice Gas Hydrodynamics in Two and Three Dimensions", Complex Syst., 1 (1987):649-707.

[9] Gunstensen, A. K., Rothman, D. H., Zaleski, S., and G. Zanetti, "Lattice Boltzmann model of immiscible fluids", Phys. Rev. A 43(8) (1991):4320-4327.

[10] Gunstensen, A. K., Ph. D. Dissertation, (1992).

[11] Chen, H., Chen, S., and W. H. Matthaeus, "Recovery of the Navier-Stokes Equations Using a Lattice Gas Boltzmann Method", Phys. Rev. A 45(8) (1991):R5339-R5341.

[12] Chen, S., Wang, J., Shan, X., and G. Doolen, "Lattice Boltzmann Computational Fluid Dynamics in Three-Dimensions", J. Stat. Phys 68(3/4) (1992):379-400.

[13] Grunau, D., Chen, S., Eggert, K. and G. Doolen, in preparation. 
[14] Chen, S. Y., Chen, H. D., Martinez, D. and W. Matthaeus, "Lattice Boltzmann Model for Simulation of Magnetohydrodynamics" Phys. Rev. Lett., 67(27) (1991):3776-3779.

[15] Bird, R. B., Stewart, W. E. and E. N. Lightfoot, Transport Phenomena, John Wiley and Sons Inc. 1960.

[16] Burgess, D. and F. Hayot, "Saffman-Taylor-Type Instability in a Lattice Gas", Phys. Rev. A. 40(9) (1989):5187-5192.; Meilburg, E. and G. M. Homsy, "Nonlinear Unstable Viscous Fingers in Hele-Shaw Flows. II. Numerical Simulation," Phys. Fluids 31 (1988):429-439.

[17] DeGregoria, A. J. and L. W. Schwartz, "A Boundary-Integral Method for TwoPhase Displacement in Hele-Shaw Cells," J. Fluid Mech. 164 (1986):383-400.

\section{Figure Captions}

Fig. 1 The theoretical prediction $(-)$ and numerical measurements $(\diamond$ and +$)$ of surface tension $\sigma$ as a function of particle density $d$ with a mass ratio $\gamma=2$.

Fig. 2 A snapshot at time $t=8300$ of the area density distribution of a spinodal decomposition simulation where the two fluids have a 10:1 mass ratio, $\gamma$. The boundaries are periodic, and the initial condition is assigned a constant density with a random color distribution. The surface tension parameter is assigned to $A=0.01$.

Fig. 3 The analytical prediction $(-)$ and direct numerical simulation $(\diamond)$ of velocity as a function of channel width $y$.

Fig. 4 The formation of a stable finger in a Hele-Shaw cell at times $t=0,2000,4000$, 6000,8000 and 10000 . A viscosity ratio of $1: 10$ is established by setting $\tau_{r}=1$ 
and $\tau_{b}=5.5$. The low-viscosity fluid (red) is penetrating the higher viscosity region (blue). 